\title{
Concept-Based Curricula
}

\section{A National Study of Critical Concepts}

\author{
Jennifer A. Brussow, MA; Karin Roberts, PhD, RN, CNE; Matthew Scaruto, MA;
}

Sheryl Sommer, PhD, RN, CNE; and Christine Mills, PhD

\begin{abstract}
As nursing education struggles to address a rapidly changing health care system, overcrowded curricula, and an increased focus on clinical reasoning skills, many programs have adopted or transitioned to concept-based curricula (CBCs), which are structured around key concepts and exemplars. Despite CBC's promised benefits, the process of developing a CBC framework may pose a challenge to programs. To address this barrier, a national study was conducted to develop a representative list of concepts and exemplars. This initiative expands on prior work by suggesting a leveled approach to positioning exemplars within a curricular sequence.
\end{abstract}

Keywords: concept-based curriculum, curriculum, educational models, nursing curriculum

Cite this article as: Brussow JA, Roberts K, Scaruto M, Sommer S, Mills C. Concept-based curricula: a national study of critical concepts. Nurse Educ. 2019;44(1):15-19. DOI: 10.1097/NNE.0000000000000515

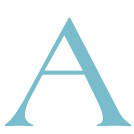

curriculum shift is underway in the field of nursing education: increasing numbers of institutions are replacing traditional practice-model curricula with concept-based curricula (CBCs). Some programs have been adopting CBCs in response to the content saturation issue plaguing nursing and the perceived disconnect between academia and practice. ${ }^{1-4}$ Nursing students are required to assimilate ever-expanding amounts of content in preparation for an increasingly complex and rapidly changing health care system, ${ }^{5}$ which is reflected in declining NCLEX pass rates. The overall first-attempt NCLEX-RN pass rate for US-educated students was $88.1 \%$ in 2006; the 2016 rate was $84.6 \% .^{6,7}$ The past decade also saw a gap emerge in pass rates for students graduating from

\footnotetext{
Author Affiliation: Research Scientist (Ms Brussow), Director of Consultation Services (Dr Roberts), Psychometrician (Mr Scaruto), VP Faculty and Institution Services (Dr Sommer), and Director of Research \& Applied Psychometrics (Dr Mills), Ascend Learning, LLC, Leawood, Kansas.

The authors are employed by Ascend Learning, whose subsidiary (Assessment Technologies Institute) produces CBC assessments. The authors' compensation is not dependent on research findings. Research design, analysis, and results reporting are conducted independently from the business unit.

Correspondence: Ms Brussow, Ascend Learning, LLC, 11161 Overbook Rd, Leawood, KS 66211 (jennifer.brussow@ascendlearning.com).

Supplemental digital content is available for this article. Direct URL citations appear in the printed text are provided in the HTML PDF versions of this article on the journal's Web site (www.nurseeducatoronline.com).

Accepted for publication: January 2, 2018

Published ahead of print: February 22, 2018

This is an open-access article distributed under the terms of the Creative Commons Attribution-Non Commercial-No Derivatives License 4.0 (CCBY-NC-ND), where it is permissible to download and share the work provided it is properly cited. The work cannot be changed in any way or used commercially without permission from the journal.

DOI: 10.1097/NNE.0000000000000515
}

BSN and associate degree in nursing (ADN) programs. In 2006, students performed comparably: $88.3 \%$ of BSN students and $88.0 \%$ of ADN passed on their initial NCLEX attempt. In $2016,87.8 \%$ of BSN students but only $81.68 \%$ of ADN students passed on their initial NCLEX attempt.

This discrepancy in pass rates may be due to the increased volume of nursing content to be covered. The Institute of Medicine recognized this issue, citing overcrowded curricula as one of the primary challenges in health care education and advising that improvements to the educational system are needed to ensure that new nurses are equipped to provide high-quality care to all patients. ${ }^{8}$ In addition, the American Association of Colleges of Nursing expressed doubt whether the current educational structure's timeframe is sufficient to adequately prepare entry-level nurses. ${ }^{9}$ In her review of curricular research efforts, Keating ${ }^{10}$ calls on the field to respond to educational changes prompted by largescale societal and systematic changes. Traditional instructorcentered approaches may no longer be adequate to deliver the amount of nursing content that is now expected to be covered.

Concept-based curricula represent a paradigm shift in nursing education, and the differences between CBCs and traditional educational models may convey several benefits to nursing programs. A shift from memorization to higherorder thinking is one of the key differences between traditional curricula and CBCs. The National League for Nursing argued that the traditional emphasis on behavioral outcomes has fostered linear thinking in nursing education and practice. ${ }^{3,11}$ In contrast, the CBC organizational framework is rooted in nursing concepts. This structure is thought to facilitate meaningful learning by emphasizing cognitive connections between newly acquired and existing knowledge. ${ }^{12,13}$ These connections have been shown to significantly 
impact nursing performance, allowing nurses to better attend to relevant information. ${ }^{2}$ Through cognitive connections and other mechanisms, CBCs are presumed to help combat content saturation by enabling students to better integrate nursing knowledge and to understand the underlying associated concepts. ${ }^{5}$

By extension, this implies that the learning facilitated by CBCs is more generalizable and less context specific, thus allowing for application of knowledge to multiple settings. Generalizable learning is consistent with the premise of a CBC: that student understanding is improved through a focus on broad organizing concepts that cross environmental settings, the life span, and health and illness. ${ }^{3}$ Conceptbased curricula's scope comprises both organizing curricula in terms of concepts and also promoting concept-based teaching. This focus on teaching conceptually also promotes an active, student-centered approach. Nursing literature has long called for a shift from educator-centered to student-centered learning because student-centered pedagogies have been shown to promote improved learning outcomes. ${ }^{1,3,14,15}$ In summary, the promise of CBCs resides in its potential to produce better-equipped entry-level practitioners with the conceptual reasoning skills required for today's increasingly complex clinical environment. Because $\mathrm{CBCs}$ have only recently begun being implemented, little efficacy research exists; however, an initial small-scale study indicates that CBCs may have beneficial effects on student retention, graduation, and NCLEX outcomes. ${ }^{16}$

Despite these purported benefits, schools may be deterred from adopting or transitioning to a $\mathrm{CBC}$ by the difficulties and challenges associated with developing such a curriculum. The extensive range of concepts in the nursing literature may prove challenging to educators attempting to select concepts during curriculum development. ${ }^{17,18}$ Programs wanting to change may also encounter faculty resistance or a lack of consensus during CBC development. ${ }^{17,19}$ A recent study cited faculty concerns about loss of control, changing role and identity, and fear of failure as the primary barriers to adopting a $\mathrm{CBC} .{ }^{20}$ To address these challenges, some programs have chosen to select concepts through a benchmarking process, which identifies and prioritizes concepts by collecting data about the concepts used in other programs. This approach benefits from providing an "objective mechanism for the decision-making process," which helps facilitate faculty agreement during the selection process. ${ }^{17(\mathrm{p} 515)}$

The benchmarking approach was adopted and expanded upon to identify the most commonly used concepts and exemplars and their curricular placement in programs across the United States. Throughout this process, concepts were defined as "organizing idea[s] or mental image [s] composed of attributes," $21(\mathrm{p} 4)$ and exemplars were defined as "examples through which the concept is contextualized." The objective of this initiative was to develop a unified set of concepts and exemplars that could be used to guide institutions' process of designing and adopting a CBC. This article details the processes used to accomplish this objective and the resulting $\mathrm{CBC}$ framework.

\section{Development}

Objectives

To facilitate the development and validation of concepts and exemplars, a task force of 5 subject matter experts was convened. A pool of candidates was created from business and professional relationships maintained by Assessment Technologies Institute. Task force members were RNs, and each held an MSN or a doctoral degree. On average, task force members had 38 years of nursing experience and 23 years of experience as a nurse educator. Task force members were program directors at nursing programs from across the United States; their nursing programs ranged in size from 30 to 250 graduates per year, with an average of 123 graduates per year. All task force members had experience with $\mathrm{CBC}$; years of experience ranged from 3 to 9 years, with an average of 4.75 years.

The task force's objectives for this initiative include the following: (1) to develop a set of concepts and exemplars representative of those used in programs across the country, (2) to review and approve rating scales for validating the concepts and exemplars and identifying appropriate curricular points for exemplar coverage, and (3) to determine the point in a CBC at which concepts and exemplars are most commonly covered and could be assessed.

\section{Process}

The initiative's process solicited feedback from several groups of subject matter experts: task force members, thought leaders, independent reviewers, and nurse educators who responded to the survey. The following 6 stages outline the general sequence of the development initiative: (1) task force convened, (2) thought leaders with experience implementing $\mathrm{CBCs}$ interviewed to gather knowledge regarding CBC, (3) preliminary list of concepts and exemplars drafted by task force, (4) independent reviewers with CBC experience reviewed and refined list of concepts and exemplars, (5) large-scale survey conducted to validate framework of concepts and exemplars and to position exemplars temporally within a curriculum, and (6) survey results reviewed by task force, and framework of concepts and temporally leveled exemplars finalized.

\section{Initial Development}

To gather preliminary information to guide the work of the task force, telephone interviews with 5 thought leaders who represented key leadership at nursing programs currently implementing a CBC were conducted. These structured interviews were conducted using a standard interview protocol designed to solicit information relating to the objectives identified above and to provide insight into existing best practices surrounding CBC implementation. Each participant received a copy of the interview protocol questions in advance of the interview, which allowed thought leaders time to reflect on their institutions' CBCs and assemble supporting information. Thought leaders were also asked to identify any sample materials they might share, such as lists of concepts and associated exemplars. 
Following the interviews and document collection, the information provided from thought leaders was collected and summarized. Results included a summary of shared and unique concepts from respondents' programs, as well as copies of curriculum-related materials provided by respondents. The task force considered this information in combination with other publicly available sets of concepts and exemplars from published articles and institutional policies to guide concept selection.

The task force then convened to draft a preliminary list of concepts and definitions based on the information assembled. Once an initial concept list was derived, breakout groups worked to draft sets of exemplars associated with each concept. Exemplars were drafted to represent illustrative issues in various settings and were selected according to incidence and prevalence. Task force members agreed that a single exemplar could appear under more than 1 concept as long as the content differed with respect to each concept (ie, the exemplar addressed different knowledge bases or nursing actions). These initial lists were reviewed following the meeting, and 2 follow-up meetings were held to integrate feedback on the drafts.

\section{Independent Review}

The resulting list of concepts and exemplars were distributed to a group of 11 independent reviewers to elicit critical review by experts who were not involved in the original development and revision of the concepts and exemplars. These reviewers were selected from candidates who were originally considered for the task force but who were unable to commit their time at that level. The original pool of candidates used to select the task force members was a nationally representative convenience sample created in partnership with Assessment Technologies Institute.

Reviewers were recruited to represent a variety of $\mathrm{CBC}$ programs and regions of the country. All reviewers held advanced degrees and were administrators and/or faculty members at nursing education programs using or transitioning to a $\mathrm{CBC}$. Independent reviewers were provided with instructions to remove, add, or edit each concept and exemplar. Concept- and exemplar-specific results were collected and incorporated into the next iteration of the concepts and exemplars over 2 follow-up task force meetings. The CBC delineation that resulted from the development process described in the steps above was comprised of 47 concepts and 290 exemplars.

\section{Validation Survey}

This preliminary list of 47 concepts and 290 exemplars was then distributed to CBC faculty and administrators via a large-scale survey. This survey was designed to collect evidence to refine and validate the concepts and exemplars. In addition, the survey gathered recommendations regarding the point within a curriculum where each concept's exemplars would be most commonly addressed. The survey began with a set of screening questions designed to ensure that respondents would be experienced with CBCs and with nurse education. Only responses from educators or administrators working in an $\mathrm{RN}$ program currently using or transitioning to a CBC program in the next 12 months were considered. In addition, respondents' institutions' CBC programs had to include a minimum of 10 concepts; preliminary discussions with the independent reviewers and task force indicated that CBCs with fewer than 10 concepts were likely to be hybrid models that still retained conventions of traditional curricular structures. Finally, respondents had to have at least 1 year of experience as a nurse educator.

The online survey was distributed to 877 nurse educators; 68 responses were collected, for a response rate of $7.8 \%$. The final set of 68 respondents included 65 individuals currently working in an $\mathrm{RN}$ program using a $\mathrm{CBC}$ and 3 working in an institution transitioning to a $\mathrm{CBC}$ in the next 3 to 6 months. The majority of respondents reported between 41 and 50 concepts in their institution's CBC. Most respondents were either nurse educators $(75 \%)$ or deans or directors of nursing $(21 \%)$. Nearly all respondents $(91 \%)$ reported that their schools were ADN programs using a CBC; $9 \%$ were BSN programs using a CBC. The majority of respondents $(79 \%)$ worked at community colleges, with the remainder divided between state institutions $(12 \%)$ and private institutions $(9 \%)$. All respondents had experience with CBC implementation: $82 \%$ of respondents had between 1 and 5 years of experience teaching in a $\mathrm{CBC}$, with an additional $10.5 \%$ having 6 or more years of experience. All respondents were experienced in nursing education: $70 \%$ of respondents had more than 5 years of teaching in nursing programs overall. Respondents represented 38 different educational institutions across 11 states distributed throughout all 5 regions of the United States.

The validation survey was composed of 3 sections: concepts, exemplars, and background information. In the first section, participants were asked for a single judgment for each of 47 concepts: "Is this concept essential to include in an ideal CBC?" This guiding question had response options of yes, no, and not sure. At the end of this section, respondents were given an opportunity to provide open-ended comments regarding any additional essential concepts they believed should be included in an ideal CBC. In the second section, participants made ratings regarding the exemplars associated with each concept. For each exemplar, participants were asked 1 yes/no question: "Is this exemplar essential to include under this concept in an ideal CBC?" They were also asked to identify the point in a CBC when the exemplar is most commonly covered: at the $25 \%, 50 \%, 75 \%$, or completion point of a nursing program. After making these ratings, participants were asked to consider whether they believed any of the exemplars for a concept belonged under a different concept and to indicate the concept under which they believed the exemplar should be placed. Participants were also given the opportunity to specify additional exemplars they believed to be essential to each concept. 
Of the 47 concepts included in the survey, 41 were rated as essential to include in an ideal CBC by $75 \%$ or more of respondents. Endorsement rates for the remaining 6 concepts (clotting, interpersonal violence, mood and affect, health policy, health care delivery systems/organizations, and health care law) ranged from $55 \%$ to $71 \%$. Four concepts (comfort, elimination, immunity, and mobility) were rated as essential to include in an ideal CBC by $100 \%$ of respondents. A similar proportion of exemplars were rated to be essential: 265 of the 290 exemplars presented in the survey were endorsed by $80 \%$ or more of respondents.

\section{Finalization}

The task force reviewed the results from the validation survey to finalize an outline for a standard $\mathrm{CBC}$. In reviewing the respondents' demographic information, the task force noted that some survey respondents were from North Carolina and Texas, which are states with a prescribed, standardized curriculum for nurse education programs using a CBC. Therefore, the task force used caution when interpreting survey results, given that multiple respondents had a similar perspective on CBCs. The task force also noted that respondents represented primarily ADN programs and discussed whether the population of respondents might proportionally underrepresent BSN programs that used a CBC. No conclusion could be drawn in the absence of national statistics regarding the number of BSN programs that use a CBC.

The task force engaged in rich discussions regarding the survey respondents' ratings and write-in responses regarding the 47 concepts. After deliberation, the group decided to eliminate the functional ability concept. Several of its exemplars could be included in the mobility concept, and the remainder could be covered in the context of other concepts. The write-in responses did not suggest that any major concept was missing from the structure. A complete list of the 46 final concepts is provided in Supplemental Digital Content, http://links.lww.com/NE/A460, Table 1.

Once the concepts were finalized, the task force worked on revising the list of exemplars. During the revision process, the task force considered ratings on the inclusion and curricular point of assessment for each exemplar, recommendations on relocating exemplars, and recommendations about additional exemplars related to specific concepts. The task force eliminated exemplars with low approval rates and combined and clarified existing exemplars as needed to ensure both clarity and completeness. The final framework included 46 concepts and 245 exemplars. Each concept is accompanied by a clear, concise definition that cites and/or adapts critical research relating to that concept where appropriate. For example, the safety concept is defined as the "minimization of risk factors that could cause injury or harm while promoting quality care and maintaining a secure environment for clients, self, and others (adapted from QSEN, 2007; NLN, 2010; Giddens, 2017).” These definitions can be used to guide course planning, instruction, and assessment efforts related to each concept.
The task force confirmed that the exemplars best conformed to a 4-level structure with respect to timing of curricular placement within a nursing education program. Each exemplar could fall at the $25 \%$ (level 1), 50\% (level 2), $75 \%$ (level 3), or completion point (level 4) within a curricular sequence. To better evaluate the survey respondents' feedback, the task force developed a set of decision rules to help determine the most appropriate placement for each exemplar within the 4-level structure where disagreement existed among respondents. These rules were as follows:

- If the difference in the percentage of respondents endorsing adjacent curricular placement levels was 5\% or less, select the higher level.

- For nonadjacent levels where the difference was $5 \%$ or less, select the higher curricular placement level. Exceptions to this rule could be made based on subject-matter expertise, considering the level for all other exemplars of the concept, and/or considering the most likely point of introduction of the concept in education programs.

- For pediatric exemplars for which there was a substantial spread in the recommended placement points (eg, congenital heart defects and sickle cell anemia), select the curricular placement level at which most programs introduce the majority of their pediatrics content (ie, level 3).

These rules were used to provide guidance for the final locations of exemplars within curricular placement levels. At times, extensive discussion occurred, especially when adjacent levels were within $6 \%$ to $10 \%$ percentage points of each other. Ultimately, the combined subject matter expertise and judgment of the task force were the deciding factor. These decisions resulted in the allocation of 66 exemplars (27\%) to level 1, 60 exemplars (24\%) to level 2, 70 exemplars $(29 \%)$ to level 3 , and 49 exemplars $(20 \%)$ to level 4. For some concepts, all associated exemplars were assigned to the same level; for example, all 4 exemplars related to acid-base balance were assigned to level 2. For other concepts, different exemplars were assigned to different levels; for example, in the mobility concept, 4 exemplars were assigned to level 2, 2 were assigned to level 3, and 1 was assigned to level 4 . As an illustrative example, Supplemental Digital Content, http://links.lww.com/NE/A461, Table 2, shows the exemplars assigned to the mobility concept.

\section{Discussion}

The final framework of 46 concepts and 245 exemplars spanning 4 levels of curricular placement represents a synthesis of information from both existing CBCs in the field and expert judgment regarding the ideal $\mathrm{CBC}$ program. The process followed by this initiative successfully met its objectives: a representative set of concepts and exemplars was developed, curricular placement levels were determined, and the resulting framework was reviewed and validated by a representative sample of nurse educators. The process information and resulting set of concepts, exemplars, and curricular placement levels provide valuable information for 
nursing education programs seeking to implement their own CBCs. Such information may help encourage adoption of $\mathrm{CBCs}$ by addressing the daunting challenge of selecting concepts from the myriad of available possibilities. The process described in this article could be followed by institutions desiring to develop their own CBC; institutions could also adapt the concept list provided here. Because the concept list included in this article was developed via a process including a diverse range of perspectives from educators across every region of the country, it may be adaptable to a wide range of programs' needs. As CBCs are increasingly adopted, a standard list of concepts would also facilitate the comparability of research on program outcomes.

\section{Limitations}

As previously noted, respondents to the validation survey primarily worked in ADN programs, so the resulting list of concepts may be more appropriate for ADN programs. In addition, some of the survey respondents worked in states with statewide CBC curricula, which may have limited the breadth of perspectives. The task force attended to this issue while interpreting survey results in order to minimize these respondents' influence on the final list of concepts and exemplars.

\section{Summary}

The process followed in this initiative combined empirical information about programs' existing CBCs with expert judgment from experienced nurse educators. Through multiple rounds of development, review, and revision, the concepts and exemplars were selected to best represent the breadth and depth necessary in a standard CBC. This initiative builds on previous $\mathrm{CBC}$ concept selection efforts by including information about the timing for each exemplar associated with a concept. The 4 levels can be used to inform placement of specific concepts and exemplars within a CBC; this level of specificity might help further decrease institutional barriers to adopting a CBC. Nurse educators and institution administrators may also use timing information to inform decisions about curricular structure or assessment cadence.

\section{References}

1. Stanley MJC, Dougherty JP. A paradigm shift in nursing education: a new model. Nurs Educ Perspect. 2010;31(6):378-380.
2. Benner P, Sutphen M, Leonard V, Day L. Educating Nurses: A Call for Radical Transformation. San Francisco, CA: Jossey-Bass; 2010.

3. Giddens JF, Brady DP. Rescuing nursing education from content saturation: the case for a concept-based curriculum. J Nurs Educ. 2007; 46(2):65-69.

4. Hardin PK, Richardson SJ. Teaching the concept curricula: theory and method. J Nurs Educ. 2012;51(3):155-159.

5. Duncan K, Schulz PS. Impact of change to a concept-based baccalaureate nursing curriculum on student and program outcomes. J Nurs Educ. 2015;54(3 suppl):S16-S20.

6. National Council of State Boards of Nursing. NCLEX® Statistics From NCSBN. Chicago, IL: NCSBN; 2006.

7. National Council of State Boards of Nursing. NCLEX® Statistics From NCSBN. Chicago, IL: NCSBN; 2016.

8. Institute of Medicine. The Future of Nursing: Leading Change, Advancing Health. Washington, DC: National Academies Press; 2010.

9. American Association of Colleges of Nursing. The Essentials of baccalaureate education for Professional Nursing Practice. Washington, DC: AACN; 1998.

10. Keating SB. Issues and challenges for nurse educators. In: SB Keating, ed. Curriculum Development and Evaluation in Nursing. Third Edition. New York: Springer Publishing Company; 2014:437-463.

11. National League for Nursing Board of Governors. Position Statement: Transforming Nursing Education. Washington, DC: NLN; 2005. Available at http://www.nln.org/docs/default-source/about/ archived-position-statements/transforming052005.pdf. Accessed December 20, 2017.

12. Abel WM, Freeze M. Evaluation of concept mapping in an associate degree nursing program. J Nurs Educ. 2006;45(9):356-364.

13. All AC, Havens RL. Cognitive/concept mapping: a teaching strategy for nursing. J Adv Nurs. 1997;25(6):1210-1219.

14. Bransford JD, Brown AL, Cocking RR (Eds). How People Learn: Brain, Mind, Experience, and School: Expanded Edition. Washington, DC: National Academies Press; 2000.

15. Getha-Eby TJ, Beery T, Xu Y, O’Brien BA. Meaningful learning: theoretical support for concept-based teaching. J Nurs Educ. 2014; 53(9):494-500.

16. Lewis LS. Outcomes of a concept-based curriculum. Teach Learn Nurs. 2014;9(2):75-79.

17. Giddens JF, Wright M, Gray I. Selecting concepts for a conceptbased curriculum: application of a benchmark approach. J Nurs Educ. 2012;51(9):511-515.

18. Pierce LNB, Laverentz D. Selecting concepts [PowerPoint slides]. Presented at the Professional Nurse Educators Group 40th Annual National Conference; Kansas City, MO; October 2013.

19. Brady D, Welborn-Brown P, Smith D, et al. Staying afloat: surviving curriculum change. Nurse Educ. 2008;33(5):198-201.

20. Hendricks SM, Wangerin V. Concept-based curriculum: changing attitudes and overcoming barriers. Nurse Educ. 2017;42(3): 138-142.

21. Giddens J, Caputi L, Rodgers BL. Mastering Concept-Based Teaching: A Guide for Nurse Educators. Elsevier/Mosby: Maryland Heights, MO; 2015. 